\title{
Atypical Presentation of Tracheobronchopathia Osteochondroplastica: Is Chronic Inflammation a Perpetrator?
}

\author{
Asmitananda Thakur ${ }^{\mathrm{a}}$ Tian Yang $^{\mathrm{a}}$ Tianjun Chen ${ }^{\mathrm{a}}$ Netra Rana ${ }^{\mathrm{c}}$ Bo Zhu ${ }^{\mathrm{a}}$ \\ Xiaohong Wei ${ }^{a}$ Lan Yang ${ }^{a}$ Guanjun Zhang ${ }^{b}$ Ming Zhang ${ }^{c}$ Mingwei Chen ${ }^{a}$ \\ Departments of ${ }^{\text {a }}$ Respiratory Medicine, ${ }^{b}$ Pathology and ${ }^{\mathrm{c}}$ Medical Imaging and Nuclear Medicine, First Affiliated \\ Hospital of Xian Jiaotong University, Xian, PR China
}

\section{Key Words}

Tracheobronchopathia osteochondroplastica · Airway inflammation · Bronchoscopy · Nodule · Ossification .

Chronic cough

\begin{abstract}
Objective: To report an atypical presentation of tracheobronchopathia osteochondroplastica (TO). Clinical Presentation and Intervention: A 59-year-old man was investigated for productive cough of 1 month. An antimycobacterial combination regime was initiated with a misdiagnosis of endobronchial tuberculosis. At follow-up, the patient reported worsening of his symptoms. CT revealed an increased intensity of the cartilage ring surrounding the trachea, and bronchoscopy showed tracheal stenosis with white, hard nodules on the airway submucosa. Histopathology confirmed the diagnosis of TO. Conclusion: This case showed that TO should be considered in patients with cough not explained by noninvasive testing and not responsive to empiric medications.
\end{abstract}

Copyright $\odot 2013$ S. Karger AG, Basel

\begin{tabular}{ll}
\hline KARGER & $\begin{array}{l}\text { ( } 2013 \text { S. Karger AG, Basel } \\
1011-7571 / 13 / 0225-0503 \$ 38.00 / 0 \quad \text { Karger }\end{array}$ \\
E-Mail karger@karger.com & $\begin{array}{l}\text { This is an Open Access article licensed under the terms } \\
\text { of the Creative Commons Attribution-NonCommercial- }\end{array}$ \\
www.karger.com/mpp & $\begin{array}{l}\text { NoDerivs 3.0 License (www.karger.com/OA-license), appli- } \\
\text { cable to the online version of the article only. Distribution } \\
\text { for non-commercial purposes only. }\end{array}$
\end{tabular}

\section{Introduction}

Tracheobronchopathia osteochondroplastica (TO) is characterized by development of multiple osseous and cartilaginous nodules in the submucosa of the trachea and the main bronchus $[1,2]$. Patients usually present with cough, recurrent respiratory tract infections, and occasionally hemoptysis $[3,4]$. TO is not usually suspected until fiber-optic bronchoscopy is performed; the bronchoscopy views together with histopathological examination of the nodules confirm the diagnosis.

\section{Case Report}

A 59-year-old male smoker (30 pack-years) was investigated for productive cough of 1 month. He had a past history of pulmonary tuberculosis ( 25 years ago; unavailable medical records) and mentioned occasional episodes of dry cough every year for the last 3 years. His family and occupational history were not significant. One month earlier, he had been diagnosed with pulmonary tuberculosis at another institution, but his sputum smear and culture 

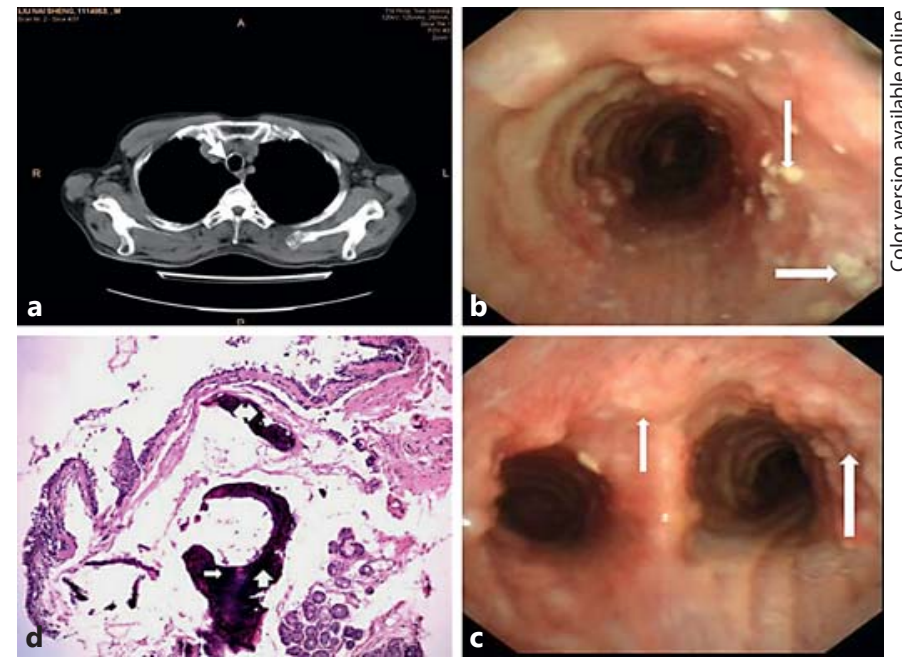

Fig. 1. a Chest CT scan showing a ring (arrow) of multiple anterolateral calcified nodules surrounding the tracheal lumen but sparing the posterior walls. b, c Bronchoscopic view of airways after 1-month therapy, showing diffuse anterolateral distribution of nodules (arrows) in the typical cobblestone appearance of TO. The posterior wall is spared. d Histopathologic view of the nodules from the patient illustrates (arrows) submucosal calcification, ossification, and cartilage formation.

were negative for acid-fast bacilli at the time of admission. His physical examination was unremarkable apart from the respiratory rate of $21 / \mathrm{min}$. Laboratory studies revealed a white blood cell count of $5.54 \times 10^{9} / 1$, platelet count of $104 \times 10^{9} / 1$, neutrophils $72.6 \%$, and an erythrocyte sedimentation rate of $21 \mathrm{~mm} / \mathrm{h}$. Liver and renal function tests were normal except for a high uric acid level $(937.9 \mu \mathrm{mol} / \mathrm{l})$. He tested negative for HIV and HBV antibodies. CT demonstrated increased density in the lateral segment of the right middle lobe, but was otherwise normal. The bronchial provocation test with methacholine was unremarkable. Bronchoscopy revealed an uneven layer of inflamed mucosa. Histopathological examination showed bronchial mucosal inflammation with squamous metaplasia but serum bone morphogenetic protein 2 was negative. An antimycobacterial combination regime (rifampicin + isoniazid + pyrizinamide + ethambutol) was initiated with a provisional diagnosis of endobronchial tuberculosis. The patient experienced relief of symptoms and was discharged, with followup scheduled after 1 month. At follow-up, the patient reported worsening cough and occasional blood-tinged sputum. CT revealed an increased density in the cartilage ring surrounding the trachea (fig. 1a). Bronchoscopy showed tracheal stenosis with white, hard spicules (fig. 1b, c). Histopathology confirmed the presence of cartilaginous and osseous nodules in the bronchial submucosa with a moderate degree of squamous metaplasia (fig. 1d). An interdepartmental consultation recommended a temporary discontinuation of the antimycobacterial combination regime (to verify that his airway changes were not drug-induced). Within 1 week of discontinuation, his symptoms eased and he had a better respiratory status. The patient was managed with broncho- scopic nodule removal and laser ablation. At the 4-month followup, radiographic findings were negative and the patient reported progressive relief in his symptoms.

\section{Discussion}

TO is limited to the large airways and does not involve the lung or other organs [1]. Changes at the mucosal surface and altered clearance of secretions result in recurrent inflammation and infection [3]. These lesions typically spread over the anterior and lateral walls of the airways (but not the posterior wall). Studies suggest that only $51 \%$ of patients with $\mathrm{TO}$ are accurately diagnosed during their lifetime [5]. Our patient was reevaluated after 1 month, $\mathrm{CT}$ and bronchoscopy revealed features suggestive of $\mathrm{TO}$, but acute deterioration is not typical of this disease [6]. Classic hypotheses include ecchondrosis and exostosis arising from the cartilaginous rings, or metaplasia of the submucosal elastic and connective tissue [1-3]. An association with lung cancer (adenocarcinoma in particular) has also been suggested [7]. Cystic fibrosis coupled with bacterial infection induces metaplastic bone replacement, as well as destruction and elimination of the bronchial cartilage. Degenerative changes in the cartilage and increased perichondrial fibrosis have been demonstrated in patients with chronic obstructive pulmonary disease and bronchial asthma. We assume that chronic inflammation of the large airway, in part due to recurrent infection, may have been the cause for our patient's initial complaint of cough. Moreover, the acute changes in the airways observed 1 month after starting the antimycobacterial combination regime and the symptomatic recovery after discontinuation of the drugs are an interesting association that remains unexplained. Whether the antimycobacterial drugs played a synergistic role by accelerating the inflammatory process is debatable. There are reports suggesting coexistence of tracheobronchial amyloidosis and TO [8]. Isolation of Klebsiella ozaenae, both in atrophic rhinitis and TO, suggests a link between these disorders [9]. Other differential diagnoses of TO include calcified lesions secondary to tuberculosis, carcinoma, papilloma, fibroma, endobronchial sarcoidosis, polychondritis, and Wegener's granulomatosis of the proximal airways. Immunohistochemical studies of TO lesions suggest a role for bone morphogenetic protein 2 [10]. The above-mentioned features were not evident in our patient, and the short history made understanding his case rather complex. Multiple factors are probably involved in the pathogenesis of TO; the cartilage ossification seen in our pa- 
tient may possibly be the result of an intense inflammatory reaction in the bronchial mucosa. More case reports and studies on the etiology of the condition will help to clarify this issue.

There is lack of consensus among clinicians on the optimum treatment, while conservative therapy aims at maintenance of airway humidity, control of infection, and avoidance of airway irritants, treatment modalities include bronchoscopy-guided excision of the nodule, laser ablation, surgical resection, and radiotherapy [1-4].

\section{Conclusion}

This case report showed that TO should be considered in patients with cough not explained by noninvasive testing and not responsive to empiric medications. CT results may be suggestive, but bronchoscopy examination, followed by histopathological findings is diagnostic of TO. Interventional bronchoscopy has an important role in the symptomatic treatment of TO.

\section{References}

$>1$ Abu-Hijleh M, Lee D, Braman SS: Tracheobronchopathia osteochondroplastica: a rare large airway disorder. Lung 2008;186:353359.

-2 Jabbardarjani HR, Radpey B, Kharabian S, et al: Tracheobronchopathia osteochondroplastica: presentation of ten cases and review of the literature. Lung 2008;186:293-297.

$\checkmark 3$ Chroneou A, Zias N, Gonzalez AV, et al: Tracheobronchopathia osteochondroplastica: an underrecognized entity? Monaldi Arch Chest Dis 2008;69:65-69.
4 Willms H, Wiechmann V, Sack U, et al: Tracheobronchopathia osteochondroplastica: a rare cause of chronic cough with haemoptysis. Cough 2008;4:4.

5 Li YY, Hu CP, Yang HZ, et al: The diagnostic value of flexible bronchoscopy in tracheobronchopathia osteochondroplastica (in Chinese). Zhonghua Jie $\mathrm{He} \mathrm{He} \mathrm{Hu} \mathrm{Xi} Z \mathrm{Za} Z$ hi 2009;32:489-492.

6 Prakash UB: Tracheobronchopathia osteochondroplastica. Semin Respir Crit Care Med 2002;23:167-175.

$>7$ Barthwal MS, Chatterji RS, Mehta A: Tracheobronchopathia osteochondroplastica. Indian J Chest Dis Allied Sci 2004;46:43-46.
Kirbas G, Dagli CE, Tanrikulu AC, et al: Unusual combination of tracheobronchopathia osteochondroplastica and AA amyloidosis. Yonsei Med J 2009;50:721-724.

$>9$ Magro P, Garand G, Cattier B, et al: Association of tracheobronchopathia osteochondroplastica and ozene (in French). Rev Mal Respir 2007;24:883-887.

-10 Tajima K, Yamakawa M, Katagiri T, et al: Immunohistochemical detection of bone morphogenetic protein-2 and transforming growth factor beta-1 in tracheopathia osteochondroplastica. Virchows Arch 1997;431: 359-363. 